\title{
Quantitative sequencing enables simultaneous absolute quantification and sequencing of environmental DNA of fish species
}

\author{
Tatsuhiko Hoshino ${ }^{1}$, Ryohei Nakao ${ }^{2}$, Toshifumi Minamoto ${ }^{3}$, and Hideyuki Doi ${ }^{4}$ \\ ${ }^{1}$ Japan Agency for Marine-Earth Science and Technology \\ ${ }^{2}$ Yamaguchi University \\ ${ }^{3}$ Kobe University \\ ${ }^{4}$ University of Hyogo
}

April 28, 2020

\begin{abstract}
Analysis of biodiversity in natural environments based on environmental DNA (eDNA) has been applied to a wide range of ecosystems and species. The combination of high-throughput sequencing technologies and eDNA analysis is a powerful tool that enables comprehensive non-invasive monitoring of species present in the environment. Quantitative data of the eDNA from each species is essential for understanding species abundance but until recently required individual assays targeting each species. Recently developed quantitative sequencing (qSeq) allows simultaneous phylogenetic identification and quantification of individual species by counting random tags added to the 5' end of the target sequence during the first DNA synthesis. Here, we applied qSeq to eDNA analysis to test its effectiveness in biodiversity monitoring. The eDNA extracted from aquaria with five fish species (Hemigrammocypris neglectus, Candidia temminckii, Oryzias latipes, Rhinogobius flumineus, and Misgurnus anguillicaudatus) across 4 days was quantified by microfluidic digital PCR using a TaqMan probe and qSeq. The eDNA abundance quantified by qSeq was consistent with dPCR for each fish species at each sampling time. However, the relative abundances of sequences obtained from high throughput sequencing did not follow the same trend as the quantitative analyses, probably due to different PCR amplification efficiencies for each species. The correlation coefficients between qSeq and dPCR were 1.052, 1.074, and 1.114 for H. neglectus, O. latipes, and M. anguillicaudatus, respectively, indicating that qSeq accurately quantifies fish eDNA. The application of qSeq to eDNA of other species will provide comprehensive quantitative data that could deepen our knowledge of natural ecosystems.
\end{abstract}

\section{Introduction}

Interest in the preservation of the environment has been heightened by environmental disruptions. Investigating biodiversity, population size, and time-course changes associated with environmental change is important for the conservation of biodiversity. Traditionally, monitoring of species has been carried out by counting individuals using distinct morphological characters. Although the direct monitoring of species is a reliable approach, it is laborious and relies on intense monitoring and sampling efforts. Moreover, certain species, such as nocturnal species, are difficult to investigate. Furthermore, direct monitoring is sometimes invasive to the environment and individual organisms.

Environmental DNA (eDNA) is a mixture of DNA released to the environment from many different species in the form of mucus, saliva, faeces, urine, gametes, and skin (Barnes and Turner, 2016; Thomsen and Willerslev, 2015; Deiner et al., 2017). By monitoring eDNA, we can monitor species in the environment without the need for sampling efforts or visual identification expertise. In the past decade, eDNA has been used to investigate the biodiversity of various species such as fish, plants, fungi, birds, and mammals (Tsuji, Takahara, Doi, Shibata, and Yamanaka, 2019). eDNA analysis is not only applied to the present environment, 
but also to studies of past biodiversity in ice cores and sediments (Parducci et al, 2017; Willerslev et al., 2007).

For ecological community analysis, DNA fragments (e.g., mtDNA and nuclear rRNA genes) used for phylogenetic identification are amplified by PCR followed by high-throughput sequencing (HTS). Species abundances in the community are often determined according to the obtained number of sequences or relative abundance of the sequences of each taxon in a sequence library. However, these methods are not quantitative because efficiencies of PCR amplification vary depending on several factors, such as amplified DNA sequences (e.g. GC content or the base adjacent to primers) and primer sequences when degenerate primers are used (Ben-Dov, Shapiro, and Kushmaro, 2012; Ruijter et al., 2009; Salipante et al., 2014; Sipos et al., 2007).

Quantitative eDNA data from each taxon is useful for estimating species biomass/abundance and determining the effects of environmental disruption. DNA quantification has been conventionally performed by quantitative PCR (qPCR) (Takahara, Minamoto, Yamanaka Doi, and Kawabata, 2012; Doi et al., 2017) and more recently by digital PCR (dPCR), which is more accurate than the former because of tolerance to PCR inhibitory substances (Hoshino and Inagaki, 2012). For quantification by qPCR or dPCR, establishing an assay for each target is needed, including the design of PCR primers, preparation of standards (if qPCR is used), and optimization of PCR conditions. Therefore, the simultaneous quantification of many species is not straightforward.

Quantitative sequencing (qSeq), which has been recently developed (Hoshino and Inagaki, 2012; Hoshino and Hamada, 2017), enables simultaneous sequencing and quantification of many species in a single HTS run. In qSeq analysis, a random sequence tag is added to the 5' end of the target sequence during single primer extension prior to PCR amplification to prepare the sequence library. If the variety of random tag sequences is sufficiently large relative to the number of targeting DNA molecules, the distribution of the random tag to DNA molecules follows the Poisson statistic. Therefore, after high-throughput sequencing, the number of DNA molecules in a sample can be estimated by counting the variety of random tags at the 5' end of the targeted sequence without being affected by PCR bias.

In this study, we applied qSeq to eDNA analysis of five species of fish in aquaria and compared the results with $\mathrm{dPCR}$ and relative quantification using conventional HTS.

\section{Materials and Methods}

\section{Aquarium experiment and sampling}

For the aquarium experiment, we examined two mock fish communities of Hemigrammocypris neglectus , Candidia temminckii ,Oryzias latipes , Rhinogobius flumineus, andMisgurnus anguillicaudatus . Mock community 1 (MC1) consisted of one individual of each of the five fish species, whereas mock community 2 (MC2) consisted of three $H$. neglectus individuals and one of each of the other four fish species (Figure 1). We used two aquaria (A and B). Each aquarium was used four times, twice for each mock community, giving two replicates (R1 and R2). This resulted in eight experimental units (2 mock fish communities $\times 2$ aquaria $\times 2$ replicates). Figure 1 shows the experimental setup used in this study.

To set up the aquaria, we added $20 \mathrm{~L}$ of tap water into the two aquaria (GEX Co. Ltd., Osaka, Japan) and heated the water with a heater (Spectrum Brands, Wisconsin, US) until the water temperature reached $25^{\circ} \mathrm{C}$. Water in the two aquaria was maintained at $25^{\circ} \mathrm{C}$ and constantly circulated with an aeration device. Before adding fish to the aquaria, water was sampled for the negative control. The first samples (Day 0) were taken an hour after adding the fish. Following this, samples was taken each day until Day 4. At sampling, two 1-L samples of surface water were collected from each aquarium. After each sample was taken, 2 L of tap water was added to each aquarium to maintain the volume of water. Fish were not fed during the 4 days. The weight of individual fish species was measured using an electronic balance immediately after the last sampling. After that, the two aquaria were bleached before being reused.

DNA extraction 
Each of the 1-L water samples was filtered immediately through a GF/F glass fibre filter (normal pore size $=0.7 \mu \mathrm{m}$; diameter $=47 \mathrm{~mm}$; GE Healthcare Japan Corporation, Tokyo, Japan). To prevent crosscontamination among the water samples, filter funnels and measuring cups were bleached after filtration. All filters were separately stored at $-20^{\circ} \mathrm{C}$ until DNA extraction. Total eDNA was extracted from each filter using a DNeasy blood and Tissue Kit (QIAGEN, Hilden, Germany) and Salivette tubes (Sarstedt AG \& Co. KG, Nümbrecht, Germany). Extraction methods were performed according to Uchii, Doi, and Minamoto (2016) with modifications. A filter sample was placed in the upper part of the Salivette tube and $220 \mu \mathrm{L}$ of solution containing $200 \mu \mathrm{L}$ Buffer AL and $20 \mu \mathrm{L}$ Proteinase $\mathrm{K}$ was added, and the tube containing the filter was incubated at $56^{\circ} \mathrm{C}$ for $30 \mathrm{~min}$. After that, the tube was centrifuged at $5000 \times \mathrm{g}$ for $3 \mathrm{~min}$, and the solution was collected in the bottom part of the tube. To increase eDNA yield, $220 \mu \mathrm{L}$ Tris-EDTA (TE) buffer was added to the filter sample and re-centrifuged at $5000 \times g$ for $1 \mathrm{~min}$. Then, $200 \mu \mathrm{L}$ of ethanol was added to the collected solution, and the mixture was transferred to a spin column. Then, total eDNA was eluted in $100 \mu \mathrm{L}$ of buffer AE following the manufacturer's instructions. All eDNA samples were stored at $-20^{\circ} \mathrm{C}$ until qSeq and $\mathrm{dPCR}$ were done.

\section{Quantitative sequencing}

Simultaneous quantification and sequencing of the extracted eDNA were performed by quantitative sequencing (qSeq) as previously described (Hoshino and Hamada, 2017; Hoshino and Inagaki, 2017). First, a single primer extension (SPE) was performed. The $20 \mu \mathrm{L}$ SPE reaction mixture consisted of $1 \times$ PrimeSTAR Max premix (Takara bio), $300 \mathrm{nM}$ qSeq-MiFish-U-F (Table 1), and $2 \mu \mathrm{L}$ of the extracted DNA. SPE was initiated by denaturation at $94^{\circ} \mathrm{C}$ for $1 \mathrm{~min}$, followed by cooling to $60^{\circ} \mathrm{C}$ at $0.3^{\circ} \mathrm{C} / \mathrm{s}$, incubation at $60^{\circ} \mathrm{C}$ for $1 \mathrm{~min}$, and final extension at $70^{\circ} \mathrm{C}$ for $10 \mathrm{~min}$. Subsequently, the excess primers that caused the incorporation of random sequence tags were completely digested by adding $4 \mu \mathrm{L}$ of Exonuclease I (Takara bio, $5 \mathrm{U} / \mu \mathrm{L}$ ) to the SPE mixture. The digestion was performed at $37^{\circ} \mathrm{C}$ for $120 \mathrm{~min}$, followed by the inactivation of Exonuclease I at $80^{\circ} \mathrm{C}$ for $30 \mathrm{~min}$. The first-round PCR mixture $(25 \mu \mathrm{L})$ contained $12.5 \mu \mathrm{L}$ of PrimeSTAR Max premix, 0.3 $\mu \mathrm{M}$ each of qSeq-MiFish-U-R and F2 primers (Table 1), and $2 \mu \mathrm{L}$ of the SPE product. After 40 cycles of amplification were performed at $98^{\circ} \mathrm{C}$ for $10 \mathrm{~s}, 55^{\circ} \mathrm{C}$ for $5 \mathrm{~s}$, and $72^{\circ} \mathrm{C}$ for $5 \mathrm{~s}$, the amplification product was subjected to agarose gel electrophoresis, and the band of the expected size was removed and purified using Nucleospin Gel and a PCR Clean-up column (Takara Bio). The qSeq-MiFish-U-R primer also contains eight $\mathrm{N}$ bases to increase the complexity for improving the sequencing quality, and thus, PhiX was not added in this study. Finally, a 2nd-round PCR was performed to add an index for Illumina sequencing as described elsewhere (Caporaso et al., 2011). The indexed PCR amplicon was purified using AMPure XP beads (Beckman Coulter, Indianapolis, IN) followed by sequencing using a MiSeq platform with MiSeq Reagent Kit v3 for 600 cycles (Illumina).

\section{Data analysis}

First, all sequences were assembled and screened by length and quality of reads using the Mother software package (v1.39.5) (Schloss et al., 2009). The processed sequence reads were classified using the MiFish pipeline (http://mitofish.aori.u-tokyo.ac.jp/mifish/). The parameters used in the MiFish pipeline analysis are described elsewhere (Sato, Miya, Fukunaga, Sado, and Iwasaki, 2018). Subsequently, representative sequences of individual operational taxonomic units (OTUs) were matched to the sequence library for picking out sequences clustered into individual OTUs using the Usearch program (http://www.drive5.com/usearch/). The random sequence tags (RST) at the end of sequences in the OTUs were counted to quantify environmental DNA from each fish species as described elsewhere (Hoshino and Hamada, 2017).

\section{Microfluidic digital PCR}

Quantification of eDNA was also performed by microfluidic digital PCR (dPCR) using the BioMark Realtime System and 12.765 Digital Array (Fluidigm Corporation, South San Francisco, CA, United States) as previously described (Hoshino and Inagaki, 2012). For each sample, $6 \mu \mathrm{L}$ of PCR mixture was prepared as follows: $3.0 \mu \mathrm{L}$ of $2 \times$ Probe qPCR mix (Takara Bio, Shiga, Japan), $0.6 \mu \mathrm{L}$ of $20 \times$ binding dye sample loading reagent (Fluidigm Corporation), $900 \mathrm{nM}$ of forward/reverse primers, $125 \mathrm{nM}$ of TaqMan probe, 
$0.015 \mu \mathrm{L}$ of ROX solution, and $1.0 \mu \mathrm{L}$ of sample DNA. We used three sets of primers and probes for the quantification of $H$. neglectus, O. latipes, and M. anguillicaudatus (Table 1). PCR was initiated at $98^{\circ} \mathrm{C}$ for $2 \mathrm{~min}$, followed by 50 cycles of $98^{\circ} \mathrm{C}$ for $10 \mathrm{~s}$ and $60^{\circ} \mathrm{C}$ for $1 \mathrm{~min}$. The amplification curves obtained from individual reaction chambers of the microfluidic chip were analysed using Fluidigm Digital PCR analysis software (Fluidigm Corporation) to obtain copy numbers.

\section{Statistical analysis}

We performed Gaussian Type II regression models with the standardized major axis method to determine the relationship between the $\log 10$ eDNA abundances obtained from qSeq and dPCR analyses with the "sma" function of the "smatr" ver. 3.4.8 package in R ver. 3.6.0 (R Core Team, 2019). For the Gaussian Type II regression model, we ignored the zero values for the modelling. We employed the Gaussian Type II model because our preliminary evaluation found higher R2 values for Type II regression models with a Gaussian distribution than with a logarithmic distribution in all cases. We compared the differences in the coefficient values by overlapping the $95 \%$ confidence interval (CI) ranges.

\section{Results}

In this study, the abundance of eDNA (i.e. the fish mitochondrial 12S rRNA gene) in the extracted DNA from the aquarium experiments using two mock fish communities was quantified with dPCR and qSeq. The eDNA of only three fish species, $H$. neglectus, M. anguillicaudatus, and O. latipes, were quantified with dPCR, whereas qSeq quantified all five fish species used in this study. We also calculated the relative abundances of the sequences of each fish species in the sequence library obtained by iTag-sequencing using Mifish primers for comparison with $\mathrm{dPCR}$ and qSeq.

We found that, for MC1 the abundance of eDNA generally decreased with time, although the variation in quantified abundance was large (Figure 2, left). Specifically, dPCR showed that the abundance of $O$. latipe $\mathrm{s}$ eDNA was 2.1-7.1 $\times 10^{5}$ (mean: $\left.4.3 \times 10^{5}\right)$ copies/L on day 0 and dropped to $5.0 \times 10^{3}-3.1 \times 10^{5}$ (mean: $\left.1.2 \times 10^{5}\right)$ copies/L on day 1 . The abundances of $H$. neglectus and $M$. anguillicaudatus on day 0 were lower than $O$. latipes, with $3.7 \times 10^{4}-1.6 \times 10^{5}$ (mean: $7.9 \times 10^{4}$ ) copies/L and $1.1 \times 10^{4}-1.2 \times 10^{5}$ (mean: 6.3 $\times 10^{4}$ ) copies/L, respectively. On the last experimental day (day 4), eDNA abundances were lowest, with mean abundances of $4.3 \times 10^{5}, 8.8 \times 10^{4}$, and $6.3 \times 10^{4}$ copies/L for O. latipes, H. neglectus, and $M$. anguillicaudatus, respectively.

Abundances obtained from qSeq were generally consistent with the results from dPCR, except for in MC1AR2. More specifically, qSeq quantified the abundance of eDNA of MC1 (Figure 2, left, middle row) on day 0 to be $1.1-5.4 \times 10^{5}$ (mean: $2.6 \times 10^{5}$ ) copies/L, $3.8 \times 10^{4}-5.8 \times 10^{5}$ (mean: $2.1 \times 10^{5}$ ) copies/L, and $1.7 \times 10^{4}-1.4 \times 10^{5}$ (mean: $\left.6.7 \times 10^{4}\right)$ copies/L for O. latipes, H. neglectus, and M. anguillicaudatus , respectively. On the last experimental day (day 4), the eDNA had decreased to $9.8 \times 10^{2}-2.9 \times 10^{4}$ (mean: $8.8 \times 10^{3}$ ) copies/L, $8.2 \times 10^{2}-6.6 \times 10^{4}$ (mean: $1.2 \times 10^{4}$ ) copies/L, and $2.2 \times 10^{3}-2.5 \times 10^{4}$ (mean: 9.5 $\times 10^{3}$ ) copies/L. In addition to the three fish species, which could be quantified using previously established assays (i.e., designing specific primers and probes), two fish species, C. temminckii and $R$. flumineus, were quantified by qSeq without establishing an assay for each species. The decreasing trend in eDNA with time was similar for these two species compared with the other three species, and the abundances were within the range of the other three species.

Mock fish community 2 had three individuals of $H$. neglectuscompared with one in MC1. Compared with $\mathrm{MC1}$, a different trend was observed in MC2, where the highest abundance of eDNA was consistently observed on day 1. After day 1, as in MC1, the abundance tended to decrease over time. The abundances of eDNA from individual species in MC2 was lower than MC1. For example, according to dPCR quantification of $\mathrm{MC} 2$ on day 0 , there were $5.0 \times 10^{3}-8.6 \times 10^{4}$ (mean: $\left.5.2 \times 10^{4}\right)$ copies/L, $3.0 \times 10^{3}-9.7 \times 10^{4}$ (mean: $\left.5.0 \times 10^{4}\right)$ copies/L, and $1.0 \times 10^{3}-1.7 \times 10^{4}$ (mean: $\left.8.3 \times 10^{3}\right)$ copies/L for O. latipes , H. neglectus, and $M$. anguillicaudatus, respectively. Similarly, qSeq resulted in fewer copy numbers of eDNA from MC1 compared with MC2, with $3.6 \times 10^{3}-5.6 \times 10^{4}$ (mean: $2.0 \times 10^{4}$ ) copies/L, $1.6 \times 10^{3}-4.9 \times 10^{4}$ (mean: 2.6 $\left.\times 10^{4}\right)$ copies/L, and $1.3 \times 10^{3}-1.7 \times 10^{4}$ (mean: $\left.8.2 \times 10^{3}\right)$ copies/L for O. latipes, H. neglectus, and $M$. 
anguillicaudatus, respectively on day 0 in $\mathrm{MC} 2$. The other two species (C. temminckii and $R$. flumineus ), which were only quantified using qSeq, showed a similar trend, with eDNA abundances peaking on day 2 and decreasing with time. Across all observations, eDNA abundances obtained from dPCR and qSeq were generally in agreement.

Although the relative proportions obtained in MiFish are not comparable with the absolute quantitative values obtained by $\mathrm{dPCR}$ or qSeq, the relative proportions in the MiFish sequencing library (Figure 2, bottom row, presented in log-scale for the comparison) followed different trends to the results from the two quantitative methods. In MC1, the MiFish results of from AR1 and AR2 were similar to the two quantitative methods (i.e., dPCR and qSeq). However, BR1 and BR2 generated completely different results; the relative abundance of eDNA of only three fish increased with time. More specifically, in MC1BR2, the relative abundance of $H$. neglectus eDNA on day 0 was $1 \%$, and it increased to $4-11 \%$ by day 4 . The relative abundances of $O$. latipes and $M$. anguillicaudatus in the sequence library also increased over time. However, these results were concurrent with a decrease in the relative abundance of eDNA from $C$. temminckii , and therefore might give a false impression that those fish species increased over time. Similarly, relative abundances in the sequence library were not consistent with the quantification results of the other two methods. For instance, in MC2AR2, the trend in relative abundance of $O$. latipes was opposite to the other two methods, with the lowest relative abundance observed in day 2 . These results confirm that relative abundances alone cannot be used to quantitatively discuss the behaviour of eDNA.

Previous studies have demonstrated a correlation between biomass and the density of eDNA in natural or laboratory environments (Yates, Fraser, and Derry, 2019; Doi et al., 2015). In this study, however, no significant correlation between biomass and abundance of eDNA was observed (Figure 3). The mean weight of $M$. anguillicaudatus was $4.2 \mathrm{~g}$, which was approximately 10 -fold higher than that of $O$. latipes at $0.47 \mathrm{~g}$ (Table 2). On the other hand, the abundance of eDNA from M. anguillicaudatus was generally lower than that from O. latipes, regardless of the quantification method (Figure 2). This discrepancy might be attributed to the difference in the discharge rate of eDNA between fish species. MC2 contained three individuals of $H$. neglectus compared with one in $\mathrm{MC} 1$; however, the abundance of $H$. neglectus $\mathrm{EDNA}$ was lower in MC2 than MC1 (Figure 3).

The abundance of eDNA for each fish species quantified with dPCR was strongly correlated with that quantified with qSeq (Figure 4). The correlations were significant $(\mathrm{p}<0001)$ and had $\mathrm{R}^{2}$ values of 0.643 , 0.859 , and 0.786 for $H$. neglactus, M. anguillicaudatus, and O. latipes, respectively. Relationships between qSeq and dPCR had slopes of $\sim 1$ and were not significantly different to $95 \%$ CIs. However, dPCR resulted in higher values than qSeq for most of the eDNA samples of O. latipes. The clear linear significant correlation between quantified abundances obtained by dPCR and qSeq indicates that using qSeq instead of the normal HTS can add quantitative information to species composition data based on obtained sequences without establishing a specific assay for each fish species of interest.

\section{Discussion}

We found that the abundance of eDNA in aquaria was highest just after fish were added and tended to decrease with time. This is consistent with a previous study which showed that the highest abundance of fish eDNA was at the start of experiments, due to the struggles during the acclimation of introduced fish to the environment (Andersen et al., 2012; Barnes et al., 2014; Takahara, Minamoto, Yamanaka Doi, and Kawabata, 2012; Jo, Murakami, Yamamoto, Masuda, and Minamoto, 2019; Sassoubre, Yamahara, Gardner, Block, and Boehm, 2016). However, we found that different mock fish communities had different peaks in eDNA abundance, with MC1 peaking on day 0 , and $\mathrm{MC} 2$ peaking on day 1 . The reason for this is not known, indicating that further studies of eDNA and fish behaviour are needed. The abundances of fish species obtained by HTS were very different from the absolute copy numbers of eDNA quantified with dPCR and qSeq. In general, PCR efficiency can affect the proportions of sequences in sequence libraries. PCR efficiency is affected by primer-target mismatches, composition (e.g., GC content) of sequences, and the bases adjacent to primers (Ben-Dov et al., 2012; Ruijter et al., 2009; Salipante et al., 2014; Sipos et al, 2007). Therefore, these factors may have influenced the proportions of sequences in our fish sequencing library, resulting in 
different proportions compared with in the original eDNA samples.

In this study, the values quantified by dPCR tended to be slightly higher than those by qSeq. It might be possible that these results are due to non-specific binding of the probes and subsequent digestion, resulting in the overestimation of dPCR. Another possible explanation might be that qSeq-MiFish-U-F did not hybridize to all target eDNA during single primer extension, causing underestimation. Indeed, the sequence of the qSeqMiFish-U-F primer used for SPE in this study has two mismatches to the sequence of O. latipes . In PCR, this mismatch resulted in low PCR efficiency; however, the mismatch disappeared once the primer synthesized template DNA because the synthesized DNA did not have a mismatch. In this study, we amplified DNA by a 50-cycle PCR, and thus the effect of the mismatch would not be significant and even DNA with two mismatch sequences was amplified and quantified. In contrast, the SPE reaction in the qSeq protocol occurs only once, and DNA is not amplified in the next PCR step if the first single-stranded DNA failed to be synthesized. Thus, since SPE would be more sensitive to mismatches in the template DNA than PCR, increasing the specificity of the SPE reaction would prevent qSeq from amplifying and quantifying environmental DNA containing mismatches.

This is the first study to employ the qSeq technique for quantifying eDNA in fish species. Using an aquarium experiment with five fish species, we demonstrated that qSeq could quantify eDNA from fish, verified by dPCR. Quantitative sequencing can simultaneously quantify multiple species by adding just one step to the standard iTag-sequencing procedure without the need to establish specific assays for individual target species like in $\mathrm{dPCR}$ or $\mathrm{qPCR}$, which require design of primers and probes, and condition optimization. Comprehensive quantitative data sets of eDNA abundance can be obtained by applying qSeq to various environments and species, providing datasets that will deepen our knowledge of natural communities and the distribution of species.

\section{Acknowledgements}

The authors are grateful for the technical assistance provided by S. Hashimoto. This work was partially supported by the Environment Research and Technology Development Fund (4-1602) by Environmental Restoration and Conservation Agency, Japan to H.D and T.M. We would like to thank Editage (www.editage.com) for English language editing.

\section{Conflict of interest}

None declared.

\section{References}

Andersen, K., Bird, K. L., Rasmussen, M., Haile, J., Breuning-Madsen, H., Kjaer, K. H., . . . Willerslev, E. (2012). Meta-barcoding of "dirt" DNA from soil reflects vertebrate biodiversity. Molecular Ecology , 21 (8), 1966-1979. doi:10.1111/j.1365-294X.2011.05261.x

Barnes, M. A., \& Turner, C. R. (2016). The ecology of environmental DNA and implications for conservation genetics. Conservation Genetics , 17 (1), 1-17. doi:10.1007/s10592-015-0775-4

Ben-Dov, E., Shapiro, O. H., \& Kushmaro, A. (2012). 'Next-base' effect on PCR amplification. Environmental Microbiology Reports , 4 (2), 183-188. doi:10.1111/j.1758-2229.2011.00318.x

Caporaso, J. G., Lauber, C. L., Walters, W. A., Berg-Lyons, D., Lozupone, C. A., Turnbaugh, P. J., . . . Knight, R. (2011). Global patterns of $16 \mathrm{~S}$ rRNA diversity at a depth of millions of sequences per sample. Proceedings of the National Academy of Sciences of the United States of America, 108, Suppl. 1, 4516-4522. doi:10.1073/pnas.1000080107

Deiner, K., Bik, H. M., Machler, E., Seymour, M., Lacoursiere-Roussel, A., Altermatt, F., . . Bernatchez, L. (2017). Environmental DNA metabarcoding: Transforming how we survey animal and plant communities.Molecular Ecology, 00 , 1-24 
Doi, H., Inui, R., Akamatsu, Y., Kanno, K., Yamanaka, H., Takahara, T., \& Minamoto, T. (2017). Environmental DNA analysis for estimating the abundance and biomass of stream fish. Freshwater Biology ,62 (1), 30-39. doi:10.1111/fwb.12846

Fukuoka, A., Takahara, T., Matsumoto, M., Ushimaru, A., \& Minamoto, T. (2016). Establishment of detection system for native rare species, Hemigrammocypris rasborella, using environmental DNA.Japanese Journal of Ecology , 66 (3), 613-620 (in Japanese).

Hoshino, T., \& Hamada, Y. (2017). Estimation of the influence of sequencing errors and distribution of random-sequence tags on quantitative sequencing. Journal of Bioscience and Bioengineering , 124 (3), 359-364. doi:10.1016/j.jbiosc.2017.04.003

Hoshino, T., \& Inagaki, F. (2012). Molecular quantification of environmental DNA using microfluidics and digital PCR. Systematic and Applied Microbiology , 35 (6), 390-395. doi:10.1016/j.syapm.2012.06.006

Hoshino, T., \& Inagaki, F. (2017). Application of stochastic labeling with random-sequence barcodes for simultaneous quantification and sequencing of environmental 16S rRNA genes. PLOS ONE ,12 (1), e0169431. doi:10.1371/journal.pone.0169431

Jo, T., Fukuoka, A., Uchida, K., Ushimaru, A., \& Minamoto, T. (2020). Multiplex real-time PCR enables the simultaneous detection of environmental DNA from freshwater fishes: A case study of three exotic and three threatened native fishes in Japan. Biological Invasions , 22 (2), 455-471. doi:10.1007/s10530-019-02102-w

Jo, T., Murakami, H., Yamamoto, S., Masuda, R., \& Minamoto, T. (2019). Effect of water temperature and fish biomass on environmental DNA shedding, degradation, and size distribution. Ecology and Evolution, 9 (3), 1135-1146. doi:10.1002/ece3.4802

Miya, M., Sato, Y., Fukunaga, T., Sado, T., Poulsen, J. Y., Sato, K., . . . Iwasaki, W. (2015). MiFish, a set of universal PCR primers for metabarcoding environmental DNA from fishes: Detection of more than 230 subtropical marine species. Royal Society Open Science,2 (7), 150088. doi:10.1098/rsos.150088

Parducci, L., Bennett, K. D., Ficetola, G. F., Alsos, I. G., Suyama, Y., Wood, J. R., \& Pedersen, M. W. (2017). Ancient plant DNA in lake sediments. New Phytologist, 214 (3), 924-942. doi:10.1111/nph.14470

Ruijter, J. M., Ramakers, C., Hoogaars, W. M. H., Karlen, Y., Bakker, O., Van den Hoff, M. J. B., \& Moorman, A. F. M. (2009). Amplification efficiency: Linking baseline and bias in the analysis of quantitative PCR data. Nucleic Acids Research, 37 (6), e45-e45. doi:10.1093/nar/gkp045

Salipante, S. J., Kawashima, T., Rosenthal, C., Hoogestraat, D. R., Cummings, L. A., Sengupta, D. J., . . . Hoffman, N. G. (2014). Performance comparison of Illumina and ion torrent next-generation sequencing platforms for 16S rRNA-based bacterial community profiling. Applied and Environmental Microbiology , 80 (24), 7583-7591. doi:10.1128/AEM.02206-14

Sassoubre, L. M., Yamahara, K. M., Gardner, L. D., Block, B. A., \& Boehm, A. B. (2016). Quantification of environmental DNA (eDNA) shedding and decay rates for three marine fish. Environmental Science and Technology , 50 (19), 10456-10464. doi:10.1021/acs.est.6b03114

Sato, Y., Miya, M., Fukunaga, T., Sado, T., \& Iwasaki, W. (2018). MitoFish and MiFish pipeline: A mitochondrial genome database of fish with an analysis pipeline for environmental DNA metabarcoding. Molecular Biology and Evolution, 35 (6), 1553-1555. doi:10.1093/molbev/msy074

Schloss, P. D., Westcott, S. L., Ryabin, T., Hall, J. R., Hartmann, M., Hollister, E. B., . . . Weber, C. F. (2009). Introducing Mothur: Open-source, platform-independent, community-supported software for describing and comparing microbial communities. Applied and Environmental Microbiology , 75 (23), 75377541. doi:10.1128/AEM.01541-09

Sipos, R., Szekely, A. J., Palatinszky, M., Revesz, S., Marialigeti, K., \& Nikolausz, M. (2007). Effect of primer mismatch, annealing temperature and PCR cycle number on 16S rRNA gene-targetting bacterial 
community analysis. FEMS Microbiology Ecology , 60 (2), 341-350. doi:10.1111/j.1574-6941.2007.00283.x

Takahara, T., Minamoto, T., Yamanaka, H., Doi, H., \& Kawabata, Z. (2012). Estimation of fish biomass using environmental DNA. PLOS ONE , 7 (4), e35868. doi:10.1371/journal.pone.0035868

Thomsen, P. F., \& Willerslev, E. (2015). Environmental DNA-an emerging tool in conservation for monitoring past and present biodiversity. Biological Conservation , 183 , 4-18. doi:10.1016/j.biocon.2014.11.019

Tsuji, S., Takahara, T., Doi, H., Shibata, N., \& Yamanaka, H. (2019). The detection of aquatic macroorganisms using environmental DNA analysis - A review of methods for collection, extraction, and detection. Environmental DNA , 1 (2), 99-108. doi:10.1002/edn3.21

Uchii, K., Doi, H., \& Minamoto, T. (2016). A novel environmental DNA approach to quantify the cryptic invasion of non-native genotypes. Molecular Ecology Resources , 16 (2), 415-422. doi:10.1111/1755-0998.12460

Willerslev, E., Cappellini, E., Boomsma, W., Nielsen, R., Hebsgaard, M. B., Brand, T. B., . . . Collins, M. J. (2007). Ancient biomolecules from deep ice cores reveal a forested southern Greenland.Science, 317 (5834), 111-114. doi:10.1126/science.1141758

Yates, M. C., Fraser, D. J., \& Derry, A. M. (2019). Meta-analysis supports further refinement of eDNA for monitoring aquatic species-specific abundance in nature. Environmental DNA , 1(1), 5-13. doi:10.1002/edn3.7

\section{Data accessibility statement}

The sequence data obtained in this study were deposited in the DDBJ database under accession number XXXXX.

\section{Author contributions}

All the authors designed the study. R.N. performed the aquarium experiment and DNA extraction. T.H. performed the quantification of eDNA using qSeq and dPCR. T.H., R.N., and H.D. analysed the data. All authors wrote the manuscript and approved the final version.

\section{Figure legends}

Figure 1. Experimental design.

Figure 2. Quantification of eDNA released from five fish species in aquaria. The left 12 plots show results from mock fish community 1, while the right 12 plots show the results from mock fish community 2 .

Figure 3. Correlation between biomass (fish weight) and the abundance of eDNA quantified by qSeq.

Figure 4. Correlation between eDNA copy number as quantified by digital PCR and quantitative sequencing (qSeq). Lines indicate regressions for each species, where pink is $H$. neglectus : $[\log (\mathrm{qSeq})=1.052(\mathrm{dPCR})$ $\left.0.043, \mathrm{R}^{2}=0.643, \mathrm{p}<0.0001\right]$; grey is $M$. anguillicaudatus $:\left[\log (\mathrm{qSeq})=1.114(\mathrm{dPCR})-0.112, \mathrm{R}^{2}=0.859\right.$, $\mathrm{p}<0.0001]$; and, orange is O. latipes : $\left[\log (\mathrm{qSeq})=1.074(\mathrm{dPCR})-0.058, \mathrm{R}^{2}=0.786, \mathrm{p}<0.0001\right]$. 


\begin{tabular}{|c|c|c|c|c|c|c|c|c|}
\hline \multicolumn{4}{|c|}{ Mock community 1 (MC1) } & \multicolumn{5}{|c|}{ Mock community 2 (MC2) } \\
\hline \multicolumn{2}{|c|}{ C. temminckii } & \multicolumn{2}{|c|}{$x 1$} & \multicolumn{3}{|c|}{ C. temminckii } & \multicolumn{2}{|c|}{$x 1$} \\
\hline \multicolumn{2}{|c|}{ H. neglectus } & \multicolumn{2}{|c|}{$x 1$} & \multicolumn{3}{|c|}{ H. neglectus } & \multicolumn{2}{|c|}{$\times 3$} \\
\hline \multicolumn{3}{|c|}{ M. anguillicaudatus } & $x 1$ & \multicolumn{3}{|c|}{ M. anguillicaudatus } & \multicolumn{2}{|c|}{$\times 1$} \\
\hline O. latipes & & \multicolumn{2}{|c|}{$x 1$} & \multicolumn{3}{|c|}{ O. latipes } & \multicolumn{2}{|c|}{$x 1$} \\
\hline \multicolumn{2}{|l|}{ R. flumineus } & \multicolumn{2}{|c|}{$x 1$} & \multicolumn{3}{|c|}{ R. flumineus } & \multicolumn{2}{|c|}{ x 1} \\
\hline Mock community & \multicolumn{4}{|c|}{ MC1 } & \multicolumn{4}{|c|}{ MC2 } \\
\hline Aquarium & \multicolumn{2}{|c|}{ A } & \multicolumn{2}{|c|}{ B } & \multicolumn{2}{|c|}{ A } & \multicolumn{2}{|c|}{ B } \\
\hline \multirow[t]{2}{*}{ Experiment } & $\mathrm{R} 1$ & $\mathrm{R} 2$ & R1 & $\mathrm{R} 2$ & $\mathrm{R} 1$ & $\mathrm{R} 2$ & R1 & $\mathrm{R} 2$ \\
\hline & day0 & day0 & day0 & day0 & day0 & day0 & day0 & day0 \\
\hline \multirow{4}{*}{$\begin{array}{l}\text { Sampling } \\
(1 L \times 2)\end{array}$} & day1 & day1 & day1 & day1 & day1 & day1 & day1 & day1 \\
\hline & day2 & day2 & day2 & day2 & day2 & day2 & day2 & day2 \\
\hline & day3 & day3 & day3 & day3 & day3 & day3 & day3 & day3 \\
\hline & day4 & day4 & day4 & day4 & day4 & day4 & day4 & day4 \\
\hline
\end{tabular}

- 2 aquaria $(A$ and $B)$ were used for each experiment.

- 2 experiments (R1 and R2) were performed for each mock community.

$\cdot 2 \times 1 \mathrm{~L}$-water samples were collected from each aquarium everyday (0-4days).

- In total, 80 water samples were filtered for eDNA extraction. 

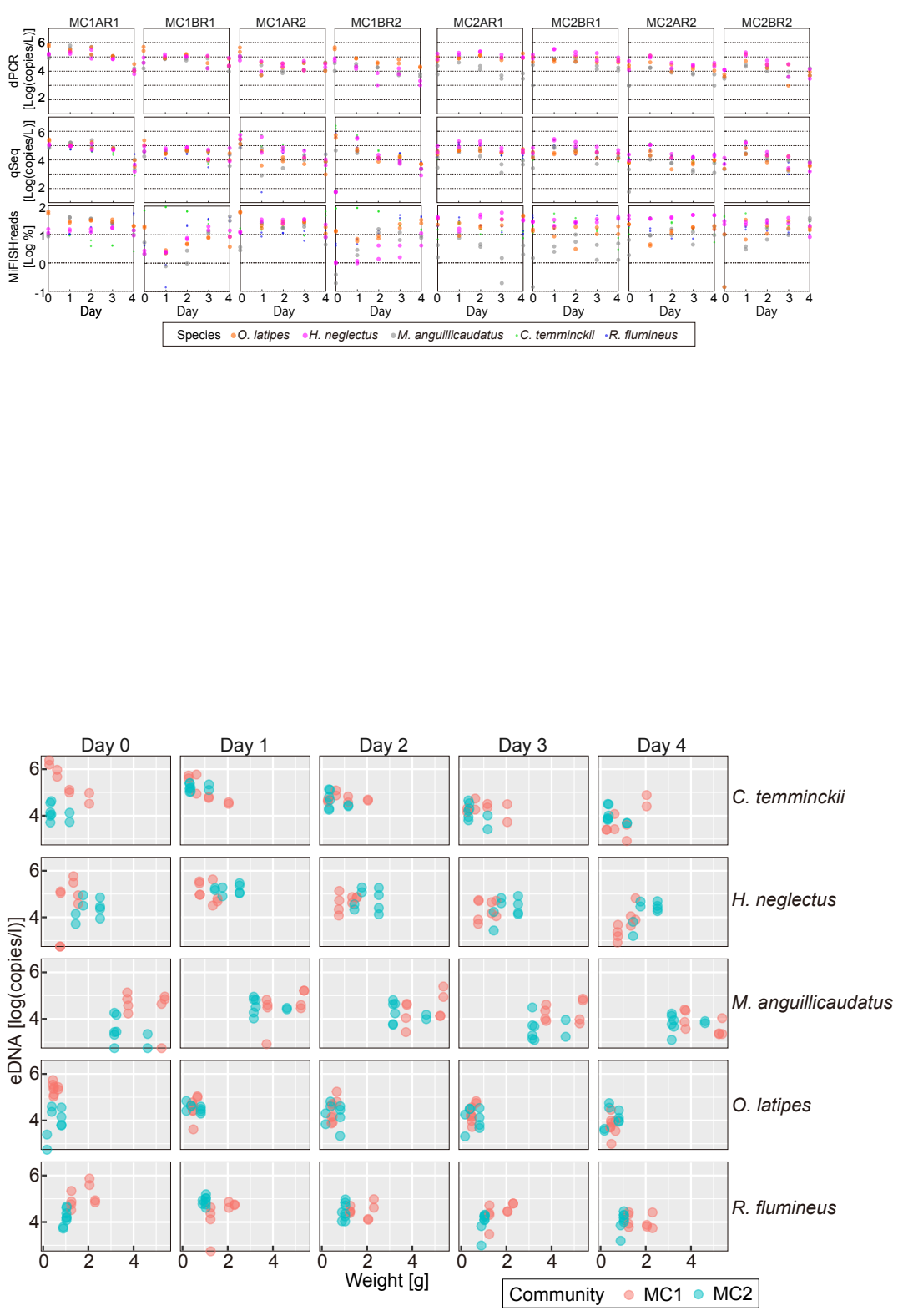


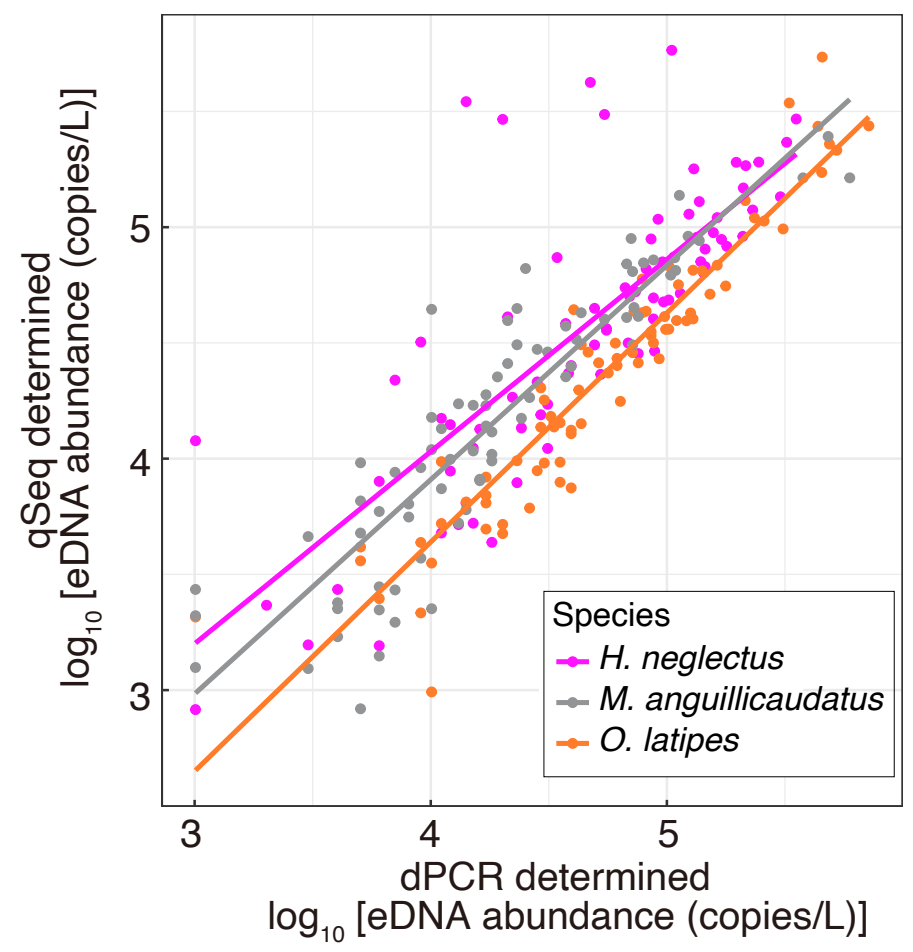

\section{Hosted file}

Table1. docx available at https://authorea.com/users/313783/articles/444214-quantitative-sequencingenables-simultaneous-absolute-quantification-and-sequencing-of-environmental-dna-of-fishspecies

\section{Hosted file}

Table2. docx available at https://authorea.com/users/313783/articles/444214-quantitative-sequencingenables-simultaneous-absolute-quantification-and-sequencing-of-environmental-dna-of-fishspecies 